\title{
Identification of two
}

\author{
Andrea Nuzzo, Andrea Negroni, Giulio Zanaroli* ${ }^{*}$ and Fabio Fava
}

\begin{abstract}
Background: Microbial reductive dechlorination of polychlorinated biphenyls (PCBs) plays a major role in detoxifying anoxic contaminated freshwater and marine sediments from PCBs. Known members of the phylum Chloroflexi are typically responsible for this activity in freshwater sediments, whereas less is known about the microorganisms responsible for this activity in marine sediments. PCB-respiring activities were detected in PCB-impacted marine sediments of the Venice Lagoon. The aim of this work was to identify the indigenous organohalide-respiring microorganisms in such environments and assess their dechlorination specificity against spiked Aroclor ${ }^{\mathrm{TM}} 1254$ PCBs under laboratory conditions resembling the in situ biogeochemistry.

Results: High PCB dechlorination activities (from $150 \pm 7$ to $380 \pm 44 \mu \mathrm{mol}$ of chlorine removed $\mathrm{kg}^{-1}$ week ${ }^{-1}$ ) were detected in three out of six sediments sampled from different locations of the lagoon. An uncultured non-Dehalococcoides phylotype of the class Dehalococcoidia closely related to Dehalobium chlorocoercia DF-1, namely phylotype VLD-1, was detected and enriched up to $10^{9} 16 \mathrm{~S}$ rRNA gene copies per gram of sediment where dechlorination activities were higher and 25-4/24-4 and 25-2/24-2/4-4 chlorobiphenyls (CB) accumulated as the main tri-/dichlorinated products. Conversely, a different phylotype closely related to the SF1/m-1 clade, namely VLD-2, also enriched highly where lower dechlorination activity and the accumulation of 25-3 CB as main tri-chlorinated product occurred, albeit in the simultaneous presence of VLD-1. Both phylotypes showed growth yields higher or comparable to known organohalide respirers and neither phylotypes enriched in sediment cultures not exhibiting dechlorination.
\end{abstract}

Conclusions: These findings confirm the presence of different PCB-respiring microorganisms in the indigenous microbial communities of Venice Lagoon sediments and relate two non-Dehalococcoides phylotypes of the class Dehalococcoidia to different PCB dechlorination rates and specificities.

Keywords: Polychlorinated biphenyls, Microbial reductive dechlorination, Organohalide respiration, Marine sediments, Dehalococcoidia

\section{Background}

Polychlorinated biphenyls (PCBs) are a family of 209 congeners composed by a biphenyl ring carrying one to ten chlorine substitutions. After more than 30 years of worldwide ban, PCBs are still widespread environmental

*Correspondence: giulio.zanaroli@unibo.it

Department of Civil, Chemical, Environmental and Materials Engineering,

University of Bologna, Via Terracini 28, 40131 Bologna, Italy contaminants [1] and are included in the list of persistent organic pollutants (POPs) targeted for elimination by the Stockholm Convention [2]. The most relevant long-term reservoirs of PCBs released into the environment are aquatic sediments, where PCBs can exert broad toxicity to wildlife [3] and enter the food chain $[1,4,5]$. Under anoxic conditions, generally occurring in such sediments few centimetres below the surface, highly chlorinated $\mathrm{PCB}$ congeners can be transformed by microbial 
activities, which sequentially remove chlorine atoms from the pollutants, making them be more susceptible to aerobic oxidative biodegradation and often less toxic and less prone to bioaccumulate than parent compounds [6, 7]. Such activity is called microbial reductive dechlorination and represents a promising process for the sustainable remediation of contaminated sediments, currently managed through expensive and highly-impacting dredging operations or capping $[8,9]$.

The occurrence of microbial PCB-dechlorinating activity in freshwater and estuarine sediments was documented in several laboratory sediment cultures and sediment-free cultures developed with synthetic media $[7,10-16]$. The process is mainly mediated by members of the phylum Chloroflexi and, less frequently, Firmicutes, which use PCBs as terminal acceptor for their electron transport chain $[7,10,17,18]$. Among the organohalide-respiring Chloroflexi, members of the genus Dehalococcoides were predominantly associated to PCB dechlorination in freshwater systems $[10,12,14,16$, $19,20]$ and more rarely in estuarine environments [21, 22]. On the other hand, Dehalococcoides-like Dehalococcoidia belonging to the o-17/DF-1 clade have been more frequently linked to PCB dechlorination activity in estuarine environments [21, 23-25], where salinity and sulfate concentrations may shift over time and space between freshwater and marine conditions [26]. Moreover, a number of pure cultures of Dehalococcoides species isolated from freshwater environments were recently shown to dechlorinate a wider range of organohalides, including PCBs $[19,27-29]$, in which some reductive dehalogenases were characterized in terms of regio specificities towards PCB congeners [27, 30]. Notably, a non-Dehalococcoides strain, Dehalobium chlorocoercia DF-1, is the sole Chloroflexi PCB respiring isolate obtained so far from estuarine sediments [24,31].

Microbial dechlorination has been less investigated in marine conditions $[17,32-38]$ where higher salinity and sulfate concentrations select for different microbial taxa compared to estuarine or freshwater environments [39]. Limited information is also available on the PCB respiring Chloroflexi in marine environments. In sediments of the Gulf of Taranto (Mar Piccolo, Ionian Sea, Italy), the enrichment of Dehalococcoides mccartyi and its PCB reductive dehalogenase-coding genes was reported during PCB dechlorination [40, 41]. Conversely, non-Dehalococcoides Dehalococcoidia were detected in sediments of the Venice Lagoon after sub-culturing of the indigenous community in the presence of exogenous PCBs [34, 42] as well as after biostimulation with zerovalent iron nanoparticles [43], suggesting that this group of organohalide respiring Chloroflexi might be relevant for the bioremediation of PCB-contaminated marine sediments. The aims of this work were: (i) to further assess the dechlorination potential, in terms of occurrence, dechlorination rate and specificity, of the indigenous microbial communities of the PCB-contaminated sediments in the Porto Marghera area (Venice Lagoon) and (ii) to assess the role of Dehalococcoidia in the process in terms of dehalogenation specificity and growth during organohalide respiration.

\section{Results and discussion PCB dechlorination}

Six different sediments from the Venice Lagoon (namely, A, B, C, D, E and F), historically contaminated by PCBs at low concentrations (Additional file 1: Table S1) were cultivated in biogeochemical conditions resembling those occurring in situ and spiked with Aroclor 1254 (see "Methods"). Neither autoclaved controls nor biologically active cultures of sediment A, B and F exhibited PCB dechlorination activities throughout incubation (31 weeks). Conversely, PCB dechlorination started after 11 weeks of incubation in sediment D cultures and after 14 weeks of incubation in sediment $C$ and $E$ cultures (Fig. 1). The onset and extent of PCB dechlorination (mol\% of $\mathrm{Cl}$ removed) was not related to the pre-existing concentration (Pearson coefficient 0.59) and distribution of congeners (Pearson coefficients in the range 0.04-0.52 for different homologue groups) of PCBs associated with the sediment matrix (Additional file 1: Table S1). This might be due to possible differences in the sediment indigenous microbial community or in some unknown sediment feature (i.e. total organic carbon available, trace metal contaminants, etc.), that might have limited the growth and activity of some fractions of the microbial community, including PCB dechlorinators. Dechlorination proceeded at maximum rates of $380 \pm 44$ and $200 \pm 31 \mu \mathrm{mol}$ of chlorine removed kg ${ }^{-1}$ week $^{-1}$ in sediment $\mathrm{D}$ and $\mathrm{C}$ cultures, respectively. Replicates of sediment $\mathrm{E}$ cultures exhibited remarkably different $\mathrm{PCB}$ dechlorination rates, being in replicate 1 (herein after referred to as sediment E1 culture) comparable to sediment $\mathrm{D}$ cultures and in replicate 2 (herein after referred to as sediment $\mathrm{E} 2$ culture) comparable to sediment $\mathrm{C}$ cultures $(325 \pm 21$ and $150 \pm 7 \mu \mathrm{mol}$ of chlorine removed $\mathrm{kg}^{-1}$ week $^{-1}$, respectively). Similarly, more extensive PCB depletions occurred in sediment D and E1 cultures ( 84 and $77 \mathrm{~mol} \%$ of penta- to octa-CBs removed, respectively), compared to sediment $C$ and E2 cultures (62 and $51 \mathrm{~mol} \%$ of penta- to octa-CBs removed, respectively).

The accumulation of several tetra- and tri-chlorinated congeners was observed in all PCB dechlorinating cultures (Fig. 2), leading to the decrease of the initial average number of chlorines per biphenyl ring (5.1) to 4.0 in sediment D and E1 cultures and to 4.4 and 4.5 in sediment $C$ and E2, respectively (Fig. 1). 


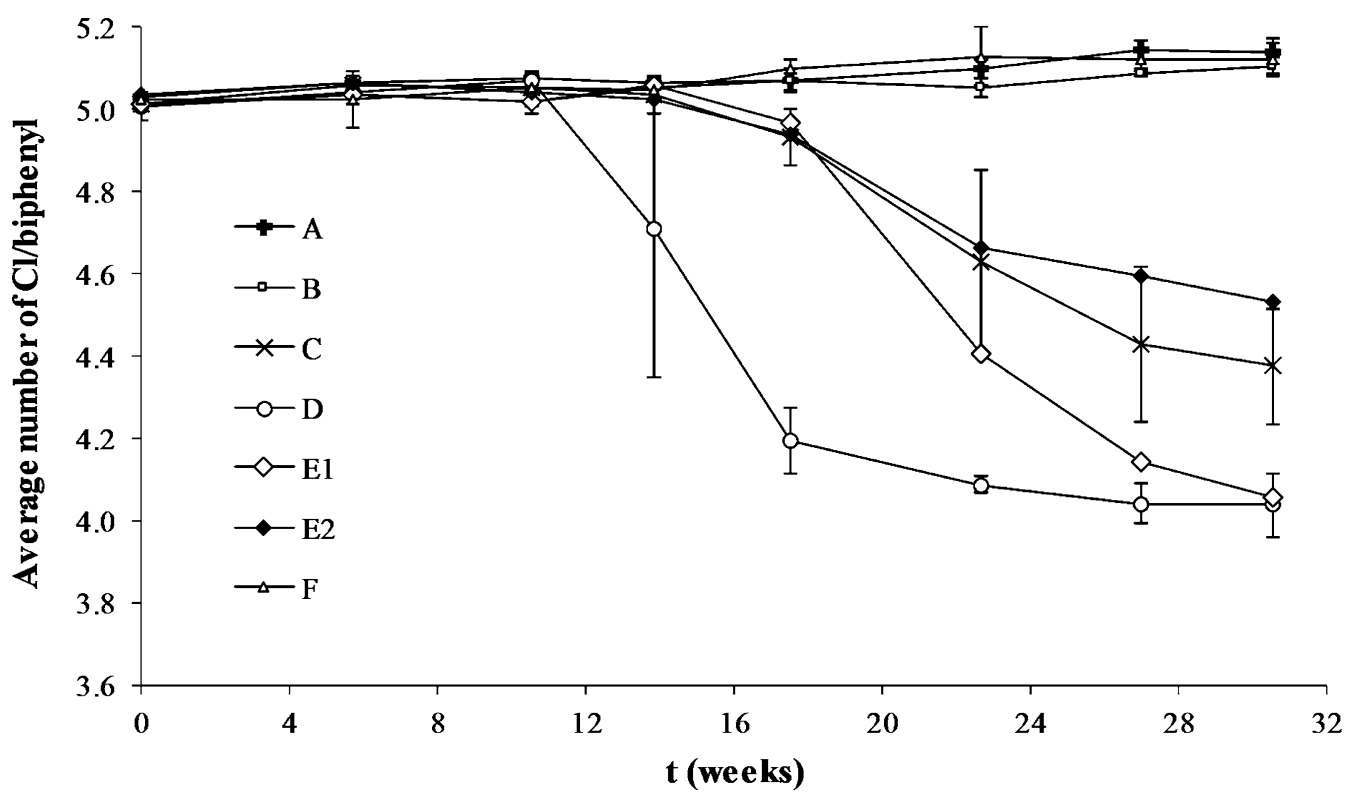

Fig. 1 Reductive dechlorination of Aroclor 1254 PCBs in the biologically active sediment cultures. Values (average number of chlorine substituents/ biphenyl molecule) are the mean ( \pm SD) of replicate cultures, except for E1 and E2 replicate cultures

The main dechlorination products that accumulated at the end of the incubation in sediment $\mathrm{D}$ were, in decreasing order of $\mu$ moles $\mathrm{kg}^{-1}$ accumulated since the beginning of incubation: 24-25 CB $\left(1074 \mu \mathrm{mol} \mathrm{kg}{ }^{-1}\right)>24-24 / 245-2 / 246-4 \mathrm{CB}$ $\left(510 \mu \mathrm{mol} \mathrm{kg}{ }^{-1}\right)>246-24 / 245-3 \mathrm{CB}\left(405 \mu \mathrm{mol} \mathrm{kg}{ }^{-1}\right)>2$ 5-4/24-4 CB $\left(356 \mu \mathrm{mol} \mathrm{kg} \mathrm{kg}^{-1}\right)>234-2 / 236-4 \mathrm{CB}$ $\left(302 \mu \mathrm{mol} \mathrm{kg}{ }^{-1}\right)>25-25 \mathrm{CB}\left(214 \mu \mathrm{mol} \mathrm{kg}{ }^{-1}\right)>25-2 / 24-$

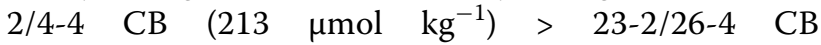
$\left(116 \mu \mathrm{mol} \mathrm{kg}^{-1}\right)$ (Fig. 2). No congener specific mass balance could be performed, due to the co-elution of several low-chlorinated products. However, considering all the possible pathways leading from the mostly depleted congeners (either eluting alone or with others, i.e., 245$34,245-25,236-34$ and 245-245/234-34 CB) to the main dechlorination products that accumulated at the end of the incubation (Fig. 2), the most recurrent dechlorination activities potentially occurring, in terms of targeted position and substitution pattern of the chlorophenyl ring, could be identified (Fig. 3a). In particular, meta dechlorination of double flanked chlorines on 2345, 2346 and 234 chlorophenyl rings appears very common to several possible pathways, along with meta and para dechlorination of single flanked chlorines in 245 and, to less extent, 34 chlorophenyl rings. Dechlorination of unflanked meta and para chlorines in 24 and 25 chlorophenyl rings also occurred, leading through different possible pathways to the accumulation of 24-2 and 25-2 congeners. This dechlorination activity was probably slower or less efficient than others, as indicated by the remarkably lower accumulation of 24-2 and 25-2 congeners compared to their parent tetra-chlorinated CBs (24-24, 24-25) and the lack of 2-2 congener accumulation. The removal of unflanked meta and para chlorines represents an important dechlorination activity to achieve extensive decontamination of sediments impacted by complex PCB mixtures, as it allows the complete elimination of meta and para chlorines, rarely observed in marine sediments but more frequently in freshwater or estuarine sediments [21, 22, 44, 45].

Conversely, 24-25 CB $\left(760 \mu \mathrm{mol} \mathrm{kg}^{-1}\right)$ and 25-25 CB $\left(511 \mu \mathrm{mol} \mathrm{kg}{ }^{-1}\right)$ were the most accumulated dechlorination products in sediment $\mathrm{C}$, where formation of 25-3 CB $\left(227 \mu \mathrm{mol} \mathrm{kg}{ }^{-1}\right)$ and 24-3 CB $\left(105 \mu \mathrm{mol} \mathrm{kg}^{-1}\right)$ was also detected, along with a much lower accumulation of 246-24/245-3 CB (117 vs $405 \mu \mathrm{mol} \mathrm{kg}{ }^{-1}$ ), 25-2/24-2/4-4 CB (100 vs $\left.213 \mu \mathrm{mol} \mathrm{kg}{ }^{-1}\right), 24-24 / 245-2 / 246-4$ CB (84 vs $510 \mu \mathrm{mol} \mathrm{kg}{ }^{-1}$ ) and $25-4 / 24-4$ CB (42 vs $\left.356 \mu \mathrm{mol} \mathrm{kg}{ }^{-1}\right)$ compared to sediment D (Fig. 2). Considering all the possible dechlorination pathways leading from the mostly depleted congeners of the Aroclor 1254 mixture (i.e., 245-25, 245-34 and 234-245/2346-34 CB) to the dechlorination products that accumulated in higher amounts (Fig. 3b), the most recurrent dechlorination activity in sediment $C$ cultures was directed towards the para position, in particular of single flanked chlorines in 34, 234 and 245 chlorophenyl rings. In addition, some meta dechlorination, mainly of single flanked chlorines in 245 chlorophenyl rings and, to less extent, of double flanked chlorines in 234 chlorophenyl rings, may have occurred 

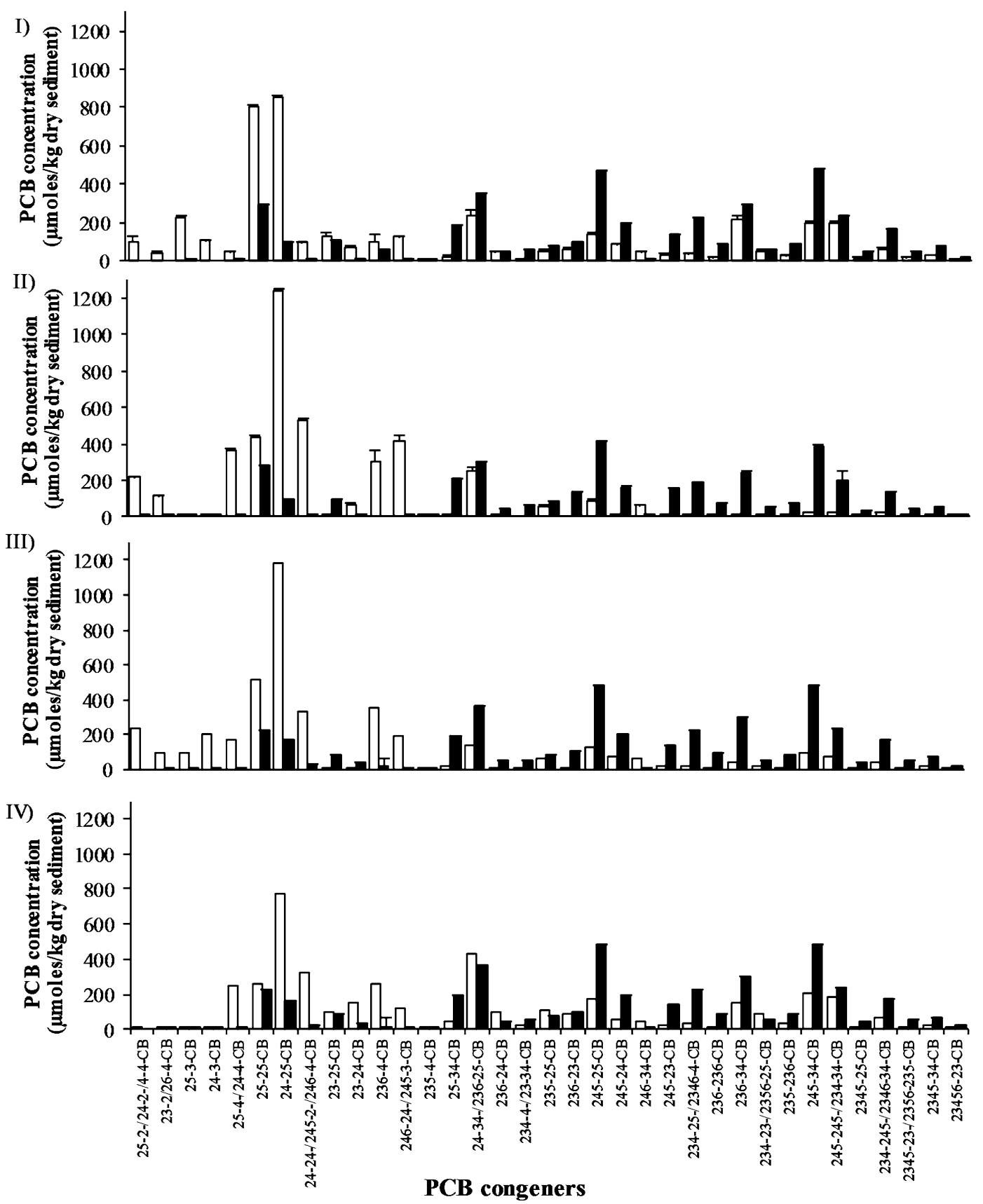

Fig. 2 Concentration ( $\mu$ moles $\mathrm{kg}^{-1}$ ) of PCB congeners in the biologically active (white bars) and sterile (black bars) sediment C, D, E1 and E2 cultures (panels I, II, III and IV, respectively) after 31 weeks of incubation. Only congeners representing more than $1 \% \mathrm{~W} / \mathrm{W}$ of the total PCBs are reported. Values are the mean $( \pm S D)$ of replicate cultures, except for E1 and E2 replicate cultures

in some dechlorination pathway (Fig. 3b). Overall, while para dechlorination of single flanked chlorines in 245 and 34 chlorophenyl rings and meta dechlorination of single flanked chlorines in 245 chlorophenyls are common to potential pathways identified in both sediment $\mathrm{C}$ and sediment $\mathrm{D}$ cultures, only potential pathways identified for sediment D culture involve also recurrent dechlorination of meta double-flanked chlorines on 2345, 2346 and 234 chlorophenyl rings and of meta and para unflanked chlorines on 24 and 25 chlorophenyl rings. These differences between sediment $\mathrm{D}$ and sediment $\mathrm{C}$ cultures suggest that $\mathrm{PCB}$ dehalogenating bacteria having 


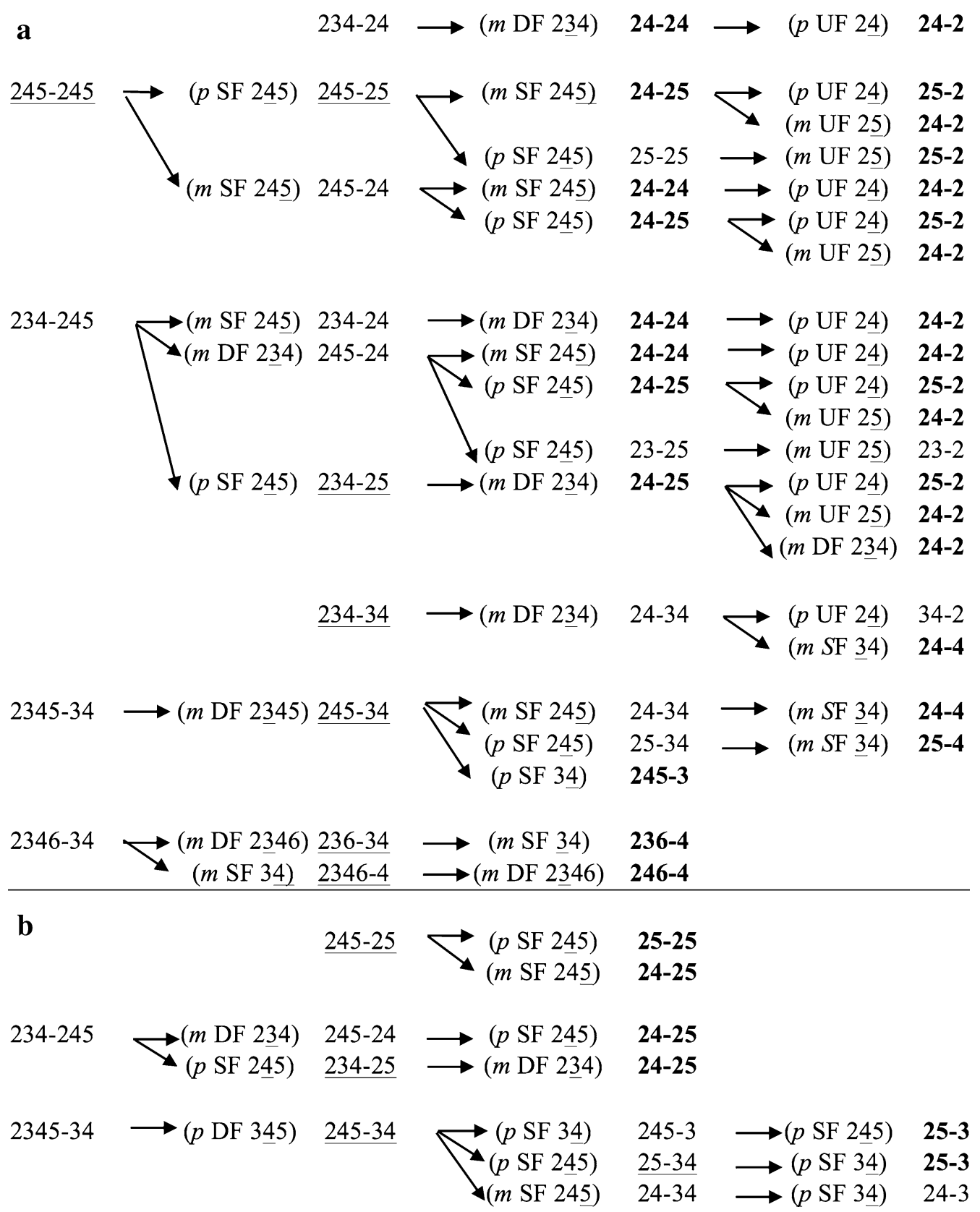

Fig. 3 Possible dechlorination patterns of the mostly depleted hexa- and penta-chlorinated congeners leading to the most prominently accumulated tetra- and tri-chlorinated congeners, in sediment D (panel a) and sediment C (pane/ b) cultures. Congeners (either eluting alone or co-eluting with others) of the original Aroclor 1254 mixture that were more extensively dechlorinated are underlined, whereas congeners (either eluting alone or co-eluting with others) that accumulated in higher amounts (more than $200 \mu \mathrm{mol} \mathrm{kg}{ }^{-1}$ after 31 weeks of incubation) are in bold characters. For each dechlorination step, the following information is reported in parenthesis: position of the removed chlorine ( $m$ : meta, $p$ : para), presence of flanking chlorines (DF double flanked, SF single flanked, UF unflanked) and chlorophenyl ring dechlorinated (removed chlorine underlined)

different dechlorination specificities were enriched in the 2 cultures.

Sediment E1 culture exhibited a dechlorination pattern similar to that of sediment $\mathrm{D}$, being 24-25 CB (879 $\left.\mu \mathrm{mol} \mathrm{kg}^{-1}\right), 25-25 \mathrm{CB}\left(514 \mu \mathrm{mol} \mathrm{kg}{ }^{-1}\right), 236-4 \mathrm{CB}$
(348 $\left.\mu \mathrm{mol} \mathrm{kg} \mathrm{kg}^{-1}\right), 25-2 / 24-2 / 4-4\left(230 \mu \mathrm{mol} \mathrm{kg}{ }^{-1}\right)$ and 24-24/245-2/246-4 CB $\left(225 \mu \mathrm{mol} \mathrm{kg}{ }^{-1}\right)$ the main dechlorination products accumulated at the end of incubation (Fig. 2). Few common features to sediment $C$ culture were however also observed, such as the detection of 24-3 CB 
(205 $\left.\mu \mathrm{mol} \mathrm{kg}^{-1}\right)$ and $25-3 \mathrm{CB}\left(88 \mu \mathrm{mol} \mathrm{kg}{ }^{-1}\right)$ (Fig. 2). A dechlorination pattern apparently similar to that of sediment D culture was observed also in sediment E2 culture, although the less extensive dechlorination observed limits the possibility to compare it with the other sediment cultures (Fig. 2).

\section{Sulfate-reducing and methanogenic activities}

Since sulfate-reducing and methanogenic bacteria are the main competitors for electron donors of organohalide respirers in anaerobic sediments, sulfate-reduction and methanogenesis were monitored over incubation to assess the presence of possible inhibitory effects of these metabolisms on the occurrence of PCB respiration. Sulfate concentrations spanned from $27 \pm 1$ to $39 \pm 1 \mathrm{mM}$ at the beginning of the incubation. Sulfate consumption was first detected after a lag phase of 6-11 weeks of incubation except in sediment $\mathrm{C}$ cultures, where it started immediately and consumed more than $40 \%$ of sulfate in the first 6 weeks (Additional file 2: Figure S1). Complete sulfate depletion was achieved in sediment $\mathrm{D}$ and sediment $\mathrm{C}$ cultures after 11 weeks of incubation, i.e. before the onset of the dechlorination process, but also in sediment $\mathrm{A}$ and $\mathrm{F}$ cultures, where no PCB dechlorination occurred (Additional file 2: Figure S1). Slower sulfate reduction took place in the non-PCB-dechlorinating culture B, where sulfate was completely reduced before week 23 , and in PCB-dechlorinating sediment E1 and E2 cultures, where complete sulfate depletion occurred at weeks 14 and 23 (Additional file 2: Figure S1). No correlation was therefore observed between the extent and rate of sulfate reduction and the occurrence, extent and rate of PCB dechlorination.

Negligible methane production (lower than $0.2 \mathrm{mmol}$ over 31 weeks of incubation) was observed in all sediment cultures, except for the non-PCB-dechlorinating cultures of sediment $\mathrm{A}$, where $56 \pm 4 \mathrm{~mL}$ (i.e. $2.5 \pm 0.2 \mathrm{mmol}$ ) of methane were produced (Additional file 3: Figure S2). Therefore, methane production was not related to the dechlorination activities detected, as previously observed in the same area [35, 42].

\section{Changes in bacterial communities and identification of $\mathrm{PCB}$ dechlorinating bacteria}

PCR followed by denaturing gradient gel electrophoresis (PCR-DGGE) of the 16S rRNA genes was carried out on sediment $\mathrm{C}, \mathrm{D}$ and $\mathrm{E}$ cultures at the beginning of incubation and during PCB dechlorination to investigate the changes of the indigenous microbial community concurrent to PCB dechlorination. Since very low methanogenic activity occurred in PCB dechlorinating cultures, the analysis was limited to Bacteria only (Fig. 4). PCRDGGE profiles were analysed to investigate changes in richness and community structure of the communities
(Fig. 4). While different trends were observed in the community richness of the dechlorinating cultures, a similar increase in the community organization index $(\mathrm{Co}$, e.g., in the Gini coefficient from approximately 30 to $50 \%$ or above) occurred after the onset of PCB dechlorination in all dechlorinating cultures. This indicates a shift of the community towards a limited number of specific phylotypes, some of which were potentially involved in the dechlorination process. Indeed, a single band (phylotype VLD-1) became prominent in sediment $\mathrm{D}$ and $\mathrm{E} 1$ cultures after the onset of PCB dechlorination, while an additional band (phylotype VLD-2) was enriched in sediment $\mathrm{C}$ and E2 cultures. The 16S rRNA gene of phylotype VLD-1 had $99.8 \%$ sequence identity (514 over 515 base pairs) to that of the uncultured bacterial clones TfC2OH76 and TfC2OH31, previously enriched in PCE dechlorinating cultures from marine tidal flat sediments [46], and $98.8 \%$ sequence identity (505 over 516 base pairs) to that of the known PCB dechlorinating Chloroflexi bacterium Dehalobium chlorocoercia DF-1 isolated from estuarine sediments [24]. Conversely, the 16S rRNA gene of phylotype VLD-2 had 100\% sequence identity (over 471 base pairs) to the uncultured Chloroflexi bacteria SF1 and $\mathrm{m}-1$, both identified as PCB dechlorinators in estuarine sediments $[21,47]$, and to phylotype VL-CHL1 (over 496 base-pairs), that was previously enriched in a PCB dechlorinating culture obtained from a sediment of the Venice Lagoon [34]. The phylogenetic placement of VLD-1 and VLD-2 within the class Dehalococcoidia of the phylum Chloroflexi is shown in Fig. 5.

PCR-DGGE analysis of the 16S rRNA genes carried out with primers specific for Chloroflexi after 27 weeks of incubation (Fig. 6) revealed the same selective enrichment of the VLD-1 and VLD-2 phylotypes in the PCB dechlorinating cultures, as well as their absence in cultures lacking PCB dechlorination activity. This confirmed that no other Chloroflexi came to relevance in any of the PCB dechlorinating cultures when the dechlorination process was almost completed (week 27) and suggested that the selective enrichment of phylotypes VLD-1 and VLD-2 is associated to the different PCB dehalogenation activities observed.

To confirm that growth of the two Chloroflexi phylotypes is linked with different dechlorination activities, qPCR was used to quantify their 16S rRNA genes and their relative abundance as a percentage the total $16 \mathrm{~S}$ rRNA genes (Table 1). Phylotypes VLD-1 and VLD-2 were present in the range of $10^{6}-10^{7}$ copies per gram of sediment in all PCB dechlorinating cultures at the beginning of the incubation, representing a minor share of the total bacterial community (typically less than 1\%), and increased throughout dechlorination. The growth yield of D. mccartyi on Aroclor 1254 PCBs in marine sediments 


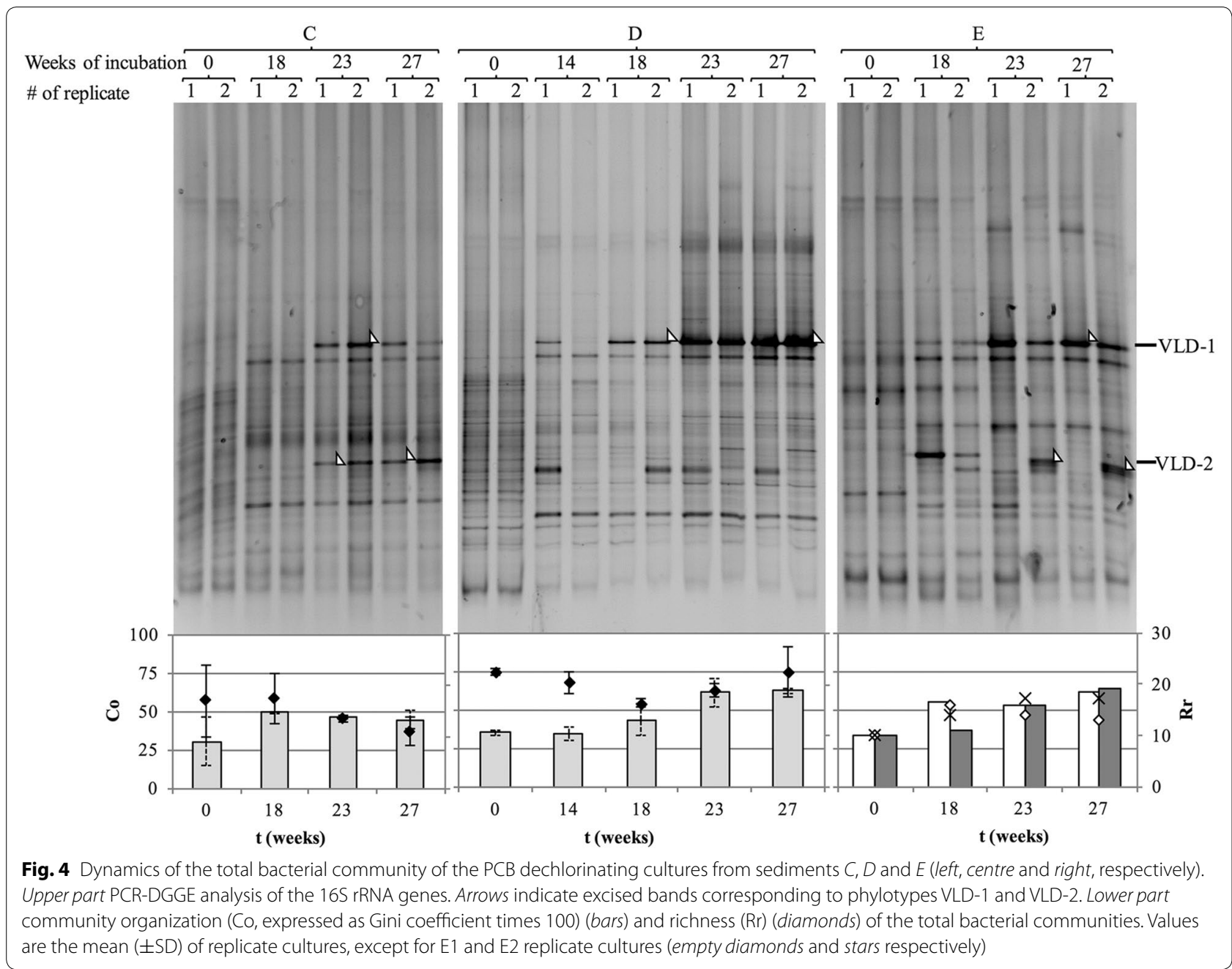

(i.e., $4.94 \times 10^{7}$ copies per $\mu \mathrm{mol} \mathrm{Cl}{ }^{-}$removed) [40], was used to calculate the expected increases in copy numbers of PCB-dechlorinating Dehalococcoidia, which were compared to the copy number increase of VLD-1 and VLD-2 phylotypes (Table 1). Remarkably, in all cultures the observed cumulative increase of VLD-1 and VLD-2 was comparable or higher than the expected one, albeit with differences between the two phylotypes, thereby providing supporting evidence that these two phylotypes grew as PCB respirers. In sediment D and E1 cultures, the 16S rRNA copy numbers of phylotype VLD-1 increased 100-fold after 27 weeks of incubation, to relative abundances of $15.2 \pm 1.0$ and $55.5 \pm 2.6 \%$, respectively. Phylotype VLD-2 16S rRNA gene copy numbers did not increase significantly in sediment $D$ cultures and eventually decreased in sediment E1 cultures after 27 weeks of incubation. On the contrary, in sediment $\mathrm{C}$ cultures, phylotype VLD-2 $16 \mathrm{~S}$ rRNA gene copy numbers increased 500-fold, up to $7.4 \times 10^{9} \pm 1.2 \times 10^{9}$ per gram of sediment after 27 weeks of incubation (accounting for
$55.9 \pm 5.2 \%$ of the bacterial $16 \mathrm{~S}$ rRNA genes). VLD-1 $16 \mathrm{~S}$ rRNA gene copy numbers also notably increased 100fold over the course of incubation, but copy numbers were more than 10 times lower than VLD-2 16S rRNA gene copies from the beginning to the end of incubation. Finally, in sediment E2 cultures, both VLD-1 and VLD-2 16S rRNA copy numbers increased from $10^{6}$ to $10^{8}$ per gram of sediment, with no significant difference between the two. Therefore, a selective enrichment of phylotype VLD-1 throughout incubation could be detected both in sediment D and E1 cultures, where phylotype VLD-2 16S rRNA gene copy numbers either remained constant or decreased. Provided that these cultures exhibited the same dechlorination specificity and rate, it is reasonable to conclude that VLD-1 was the predominant organohalide respirer in these cultures and could thus be linked to the more rapid and extensive dechlorination activity involving meta double flanked chlorines, meta and para single flanked chlorines and, to less extent, unflanked meta and para chlorine (Fig. 3a). The enrichment of 


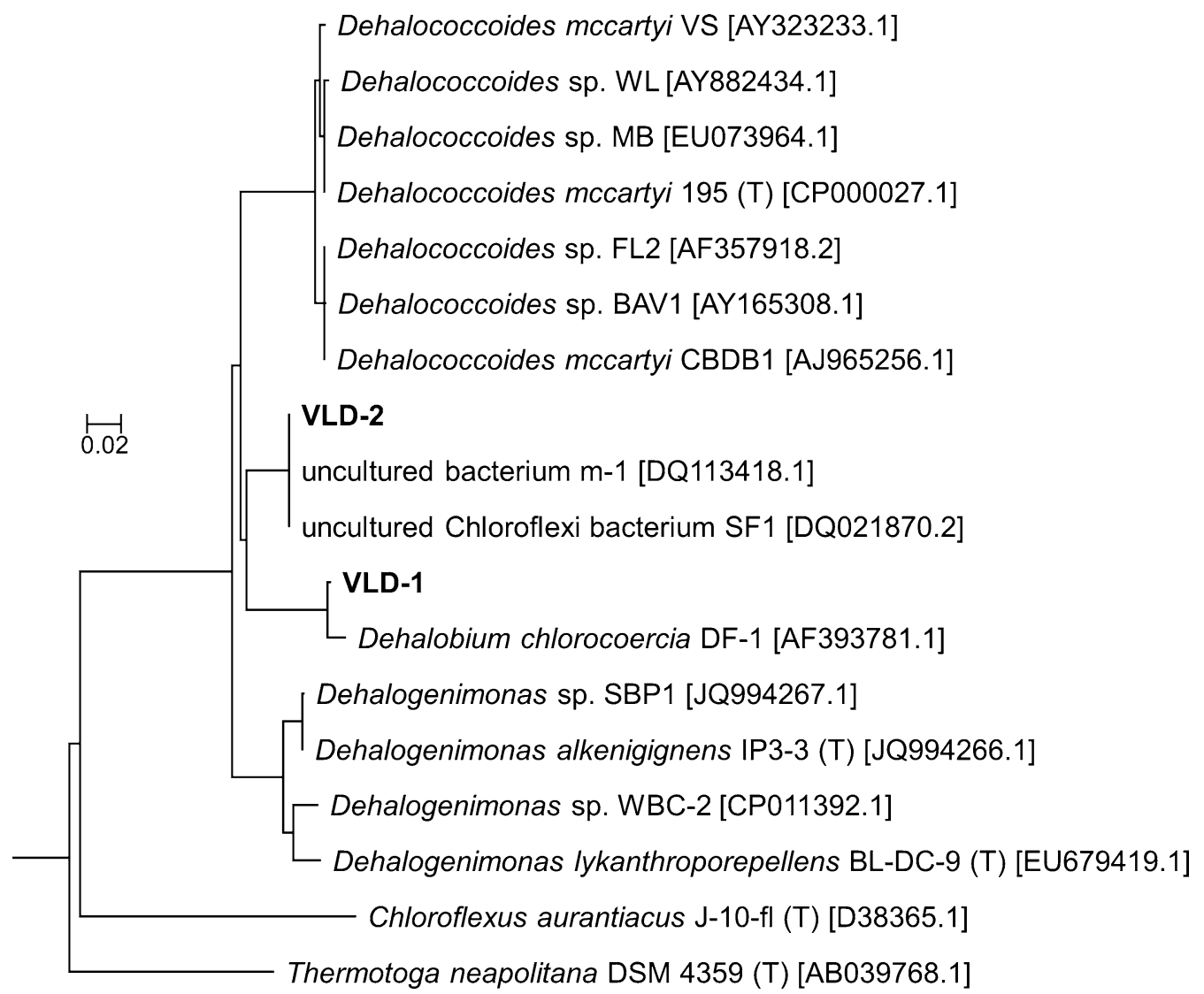

Fig. 5 Phylogenetic placement of VLD-1 and VLD-2 within the class Dehalococcoidia of the phylum Chloroflexi. The tree was created using the Tree Builder tool based on the Neighbour Joining method available at the Ribosomal Database Project (RDP, release 11, http://rdp.cme.msu.edu)

VLD-2 phylotype in sediment $\mathrm{C}$ and E2 cultures was less selective, since in both cases it was accompanied by a simultaneous enrichment of phylotype VLD-1. However, VLD-2 copy numbers in sediment $C$ cultures were always more than tenfold higher than VLD-1 copy numbers, indicating that it nevertheless constituted a major share of the dehalogenating bacteria, whereas similar initial and final copy numbers were observed for VLD-1 and VLD-2 in sediment E2. Therefore, the peculiar features of the dechlorination activities exhibited by sediment $\mathrm{C}$ cultures, which differ from sediment D and E1 cultures (i.e. single or double flanked para chlorines and, to less extent, single flanked meta chlorines removal) (Fig. 3b), could be ascribed to phylotype VLD-2. The presence of some meta dechlorination of double flanked chlorines in sediment $C$ culture, on the other hand (Fig. 2a), could be explained by the concurrent, though more limited, enrichment of VLD-1 phylotype. The concomitant and equivalent enrichment of VLD-1 and VLD-2 phylotypes in sediment E2 cultures is consistent with the observation of a dechlorination pattern that is partially similar to that of sediment D and E1 cultures and partially to that of sediment $\mathrm{C}$ cultures. Finally, both PCB-dechlorinating phylotypes occurred at very low relative abundances in all cultures at the beginning of incubation, but their differential enrichment and the subsequent different dechlorination activity exhibited suggests that some unknown features of the sediment, or differences in the non-organohalide-respiring fraction of their indigenous microbial community, might preferentially favour the growth of one PCB respiring bacterium over the other. These findings overall confirm the role of non-Dehalococcoides Dehalococcoidia in the reductive dechlorination of PCBs in marine sediments of Venice Lagoon, further supporting the relevance of this group of microorganisms for the bioremediation of marine environments contaminated by organohalides.

\section{Conclusions}

Three out of six marine sediments used in the study showed PCB-dechlorination activities under laboratory conditions resembling the in situ biogeochemistry, suggesting that a potential for dehalogenation is present, although not ubiquitously, in the microbial communities 


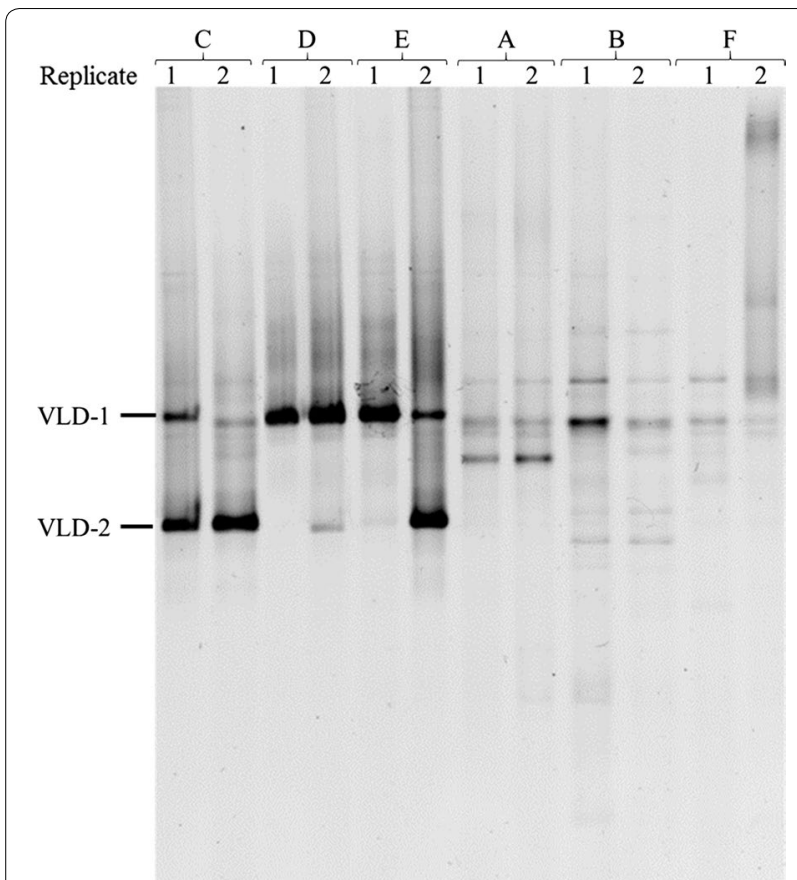

Fig. 6 PCR-DGGE analysis of the Chloroflexi bacterial community after 27 weeks of incubation in the PCB dechlorinating $(C, D, E)$ and non-dechlorinating cultures $(A, B, F)$. Arrows indicate excised bands corresponding to phylotypes VLD-1 and VLD-2

of the Porto Marghera area of Venice Lagoon. Two nonDehalococcoides phylotypes of Dehalcoccoidia, closely related to other PCB-respiring microorganisms previously identified in estuarine sediments, were associated to two distinct dechlorination activities. Among these, phylotype VLD-1 is capable of unflanked meta and para chlorines removal, and thus potentially able to achieve extensive decontamination and detoxification of sediments impacted by complex PCB mixtures. These nonDehalococcoides Dehalococcoidia are therefore candidate targets for further enrichment and isolation efforts, aiming at the production of PCB dechlorinating inocula for bioaugmentation purposes, and/or for biostimulation approaches to promote the decontamination of sediments in the Venice Lagoon and other PCB impacted marine areas.

\section{Methods}

\section{Venice Lagoon sediments}

Six sediments (A, B, C, D, E, and F) from different locations of the first industrial area of Porto Marghera (Venice Lagoon, Italy) were used in this study, along with the seawater collected from the same area. Sediments were impacted by PCBs in the range 0.2 (sediment F) -3.3 (sediment D) $\mathrm{mg} \mathrm{kg}^{-1}$ consisting predominantly of highly chlorinated congeners (i.e. sediments B and D) but also with considerable percentages of medium-low chlorinated ones (i.e., sediment A) (Additional file 1: Table S1).

\section{Preparation and sampling of sediment cultures}

A set of four $100 \mathrm{~mL}$ anaerobic slurry cultures (duplicate biologically active and sterile controls) was prepared for each sediment anaerobically using site water for sediment re-suspension ( $20 \%$ dry w/v) under nitrogen atmosphere [33]. Given the limited number and low concentration of PCB congeners occurring in each sediment (Additional file 1: Table S1), sediment cultures were spiked with Aroclor $1254\left(20 \mathrm{~g} \mathrm{~L}^{-1}\right.$ stock solution in acetone $)$ at a final concentration of $1 \mathrm{~g}$ of $\mathrm{PCBs} \mathrm{kg} \mathrm{k}^{-1}$, to favour the

Table 1 16S rRNA gene copy numbers and relative abundance of phylotypes VLD-1 and VLD-2

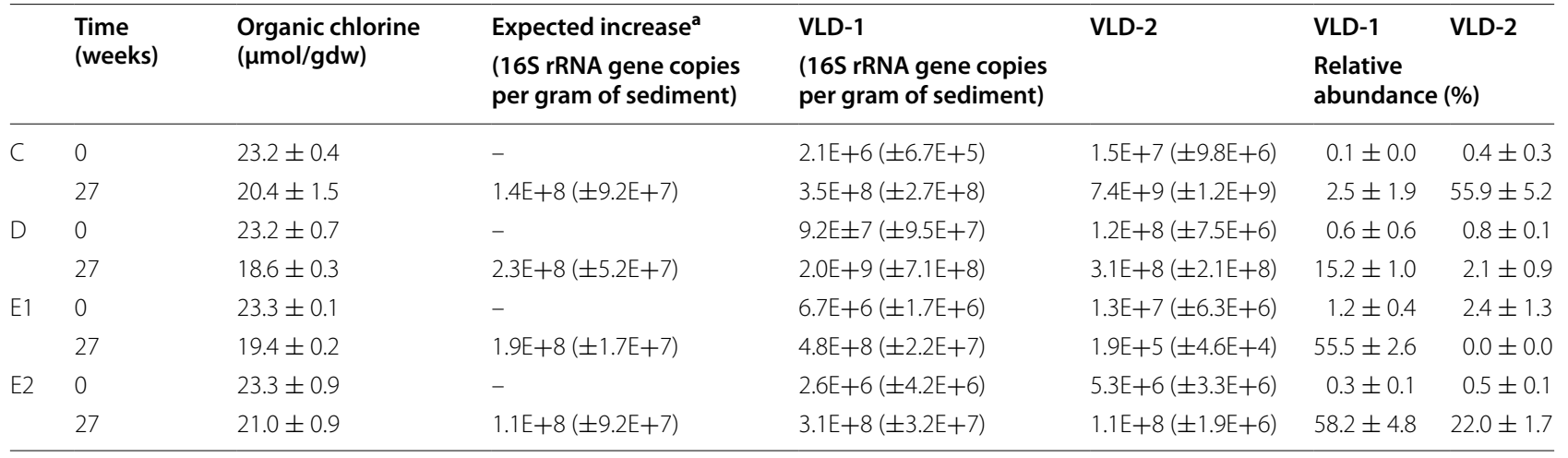

Organic chlorine, expected increase of $16 \mathrm{~S}$ rRNA gene copies of PCB respirers, observed $16 \mathrm{~S}$ rRNA genes copy numbers per gram of sediment and relative abundance (vs total number of bacterial 16S rRNA genes) of phylotypes VLD-1 and VLD-2 at the beginning and after 27 weeks of incubation (27 week). Values are the mean ( \pm SD) of triplicate analysis performed on metagenomic DNA extracted from each replicate culture with primer pairs targeting 16S rRNA genes of individual phylotypes (VLD-1, VLD-2) and total bacteria

a The expected increase of 165 rRNA gene copy numbers of PCB respirers was calculated from the organic chlorine removed over incubation using the growth yield of D. mccartyi on PCBs reported in Matturro et al. [40] 
enrichment of sediment indigenous PCB-respiring bacteria and better assess their PCB dechlorination potential. Sediment cultures were incubated statically in the dark at $28{ }^{\circ} \mathrm{C}$ for 31 weeks and periodically sampled according to the procedure described by [12] to analyse: (i) the volume and composition of the biogas (i.e., methane and $\mathrm{CO}_{2}$ ), (ii) the congeners and concentration of PCBs in the sediment, (iii) the concentration of sulfate in the water phase and (iv) the structure and composition of the microbial community.

\section{PCB extraction and analytical procedures}

PCBs were batch extracted in duplicate from each replicate culture according to procedures described elsewhere [33]. GC-ECD analyses of extracted PCBs were performed under the analytical conditions described in literature [32]. Qualitative analysis was performed by comparing retention times (relative to octachloronaphtalene) of parent PCBs and their dechlorination products with those of PCBs occurring in Aroclor 1242 and Aroclor 1254 standard mixtures (Ultra Scientific Italia, Bologna). Quantitative analyses were performed using the GC-ECD response factor of each $\mathrm{PCB}$, obtained from linear five-points calibration curves Aroclors (in the range $1.0-50.0 \mathrm{mg} \mathrm{L}^{-1}$ ) and the weight percentage of each congener occurring in Aroclors reported elsewhere [48]. PCB concentrations ( $\mu$ moles $\mathrm{kg}^{-1}$, referred to the sediment dry weight), the average number of $\mathrm{Cl}$ per biphenyl and dechlorination rates ( $\mu$ moles of $\mathrm{Cl}$ released $\mathrm{kg}^{-1}$ week ${ }^{-1}$, referred to the sediment dry weight) were calculated assuming co-eluting congeners to be present in equal proportions as described in previous works [42].

Biogas production was measured at each sampling with an airtight glass syringe, while its composition was determined via $\mu$ GC-TCD as described previously [49]. Sulfate concentration in the water phase was determined with IC-CD as described in [32].

\section{Community analysis by PCR-DGGE of the $16 \mathrm{~S}$ rRNA gene}

Total DNA was extracted from the wet sediment (approximately $250 \mathrm{mg}$ ) recovered from the centrifugation of $2 \mathrm{~mL}$ slurry samples at $10,000 \times \mathrm{g}$ for $10 \mathrm{~min}$ with the UltraClean Soil DNA kit (MoBio Laboratories, Carlsbad, CA, USA) according to the protocol "for maximum yields" provided by the manufacturer preceded by treatment with Proteinase $\mathrm{K}$ and Lysozyme as described elsewhere [42]. Total DNA was quantified using Qubit ${ }^{\circledR}$ dsDNA HS Assay Kit with a Qubit 3.0 fluorimeter, following the manufacturer's specifications.

For DGGE analysis, 16S rRNA genes of the bacterial community were PCR-amplified with the GC-clamped primer GC-357f and primer 907r [39], while 16S rRNA genes of putative dechlorinating Chloroflexi were PCR amplified with the GC-clamped primer GC-348f and primer 844r [47]. PCR reactions were performed as described in previous works [27]. DGGE of PCR products (approximately $400 \mathrm{ng}$ DNA per lane) were performed in $7 \%(\mathrm{w} / \mathrm{v})$ polyacrylamide gels at $55 \mathrm{~V}$ for $16 \mathrm{~h}$. Denaturing gradients from 40 to $60 \%$ denaturant and from 45 to $55 \%$ denaturant were used to resolve total Bacteria and Chloroflexi-specific amplicons, respectively. Digital images of gels were captured in UV transillumination after staining with SYBR Green I.

Community richness $(\mathrm{Rr})$ and community organization (Co) indexes were calculated from DGGE image analysis as described in literature [50-52]. In particular, the range-weighted richness was calculated from the total number of bands in the pattern and the denaturing gradient comprised between the first and the last band of the pattern, whereas the community organization was derived from Pareto-Lorenz (PL) evenness curves and the respective Gini coefficient.

Most prominent DGGE gel bands were cut, DNA eluted overnight at $4{ }^{\circ} \mathrm{C}$ in sterile water, re-amplified and resolved again in DGGE as described above, before amplification with non-GC-clamped primers. Amplicons were finally purified in the presence of $10 \mathrm{U}$ of ExoI and $1 \mathrm{U}$ of FastAP enzymes (Thermo Scientific Italia s.r.l, Milan, Italy) at $37{ }^{\circ} \mathrm{C}$ for $15 \mathrm{~min}$ before sequencing with the corresponding forward primer. Sequencing was performed by BMR Genomics (Padova, Italy). Each 16S rRNA gene sequence obtained ( $\sim 500 \mathrm{bp}$ ) was aligned to the bacterial 16S rRNA database of the Ribosomal Database Project (RDP, release 11, http://rdp.cme.msu.edu) and the closest relative and closest cultured relative retrieved with the Seqmatch tool. The phylogenetic affiliation of each sequence was obtained from the same website with the Classifier tool. Nucleotide sequences were deposited in the GenBank database under the Accession Numbers KR013281 and KR013282.

\section{Quantification of target Dehalococcoidia by qPCR}

Primer pairs were designed for the specific amplification of two partial 16S rRNA gene sequences detected in the PCB dechlorinating cultures by DGGE using the bacterial primers pair 357f/907r (see Results section). Primers were designed with the Primer-BLAST website (http:// www.ncbi.nlm.nih.gov/tools/primer-blast) [53], using each target sequence as PCR template, a primer melting temperature from 58 to $61^{\circ} \mathrm{C}$, the Refseq RNA database for specificity checking, PCR product Tm between 80 and $90{ }^{\circ} \mathrm{C}$, optimum at $80{ }^{\circ} \mathrm{C}$. The specificity of each candidate primer pairs was checked in silico with the ProbeMatch tool available at the RDP II web site; primer pairs having the highest in silico specificity were checked 
for cross-specificity in vitro in end-point PCR and qPCR assays (see details below).

The gene copies of the target Chloroflexi phylotypes and the total bacterial 16S rRNA genes were quantified through qPCR using a StepOne ${ }^{\mathrm{TM}}$ Real-Time PCR System (Applied Biosystems, Monza, Italy) and StepOne software $\mathrm{v} 2.0$ according to the manufacturer instructions. Designed primer pairs used to target selected Chloroflexi were: (i) 682f (5'-AGGCGAAAGCGGTTTCCAA-3') and $814 \mathrm{r}$ (5'-ACTTAAAGCGTTAGCTTCGGCA-3') for VLD-1; (ii) 585f (5'- TCAACTGGGAGGAGTCATTCG-3') and 697r (5'- GAAACAGCCTAGAAAACCGCC-3') for VLD-2 (Additional file 4: Table S2). Finally, known primers 920f [54] and 1044r [55] were used to target bacterial 16S rRNA genes. PCR cycles were as follows: (i) $95{ }^{\circ} \mathrm{C}$ for $10 \mathrm{~min}$, (ii) 40 cycles of denaturing at $95{ }^{\circ} \mathrm{C}$ for $30 \mathrm{~s}$, annealing at 56,59 or $54{ }^{\circ} \mathrm{C}$ for $30 \mathrm{~s}$ (with uncultured Chloroflexi VLD-1, uncultured Chloroflexi VLD-2 and total bacterial 16S rRNA gene primer pairs, respectively), and elongation at $72{ }^{\circ} \mathrm{C}$ for 30 secs, (iii) denaturation at $95^{\circ} \mathrm{C}$ for $15 \mathrm{~s}$ followed by a melting curve from 60 to $95{ }^{\circ} \mathrm{C}$ and fluorescence measure every $0.3{ }^{\circ} \mathrm{C}$. The qPCR reactions $(25 \mu \mathrm{L})$ were set-up as follows: $1 \times$ Power SYBR ${ }^{\circledR}$ Green PCR Master Mix (Applied Biosystems, Monza, Italy), forward and reverse primers at $350 \mathrm{nM}$ each and $2.5 \mu \mathrm{L}$ of DNA template. 5-point standard curves were included in each plate from $6.8 \times 10^{3}$ to $6.8 \times 10^{8}$ copy numbers using PCR products of DGGE gel-purified bands as standard template for Chloroflexispecific targets or of $E$. coli $16 \mathrm{~S}$ rRNA gene for total bacteria. DNA was purified with Wizard ${ }^{\circledR}$ SV Gel and PCR Clean-Up System (Promega Italia S.r.l, Milano, Italy) according to the manufacturer protocol and quantified with a P330 Nanophotometer (Implen GmbH, Munich, Germany). Amplification efficiencies ranged from 85 to $106 \%$, with $R^{2}=96-100 \%$. Samples and standards were set up in triplicate reactions. Genes copy numbers per gram of sediment were calculated and relative abundance was determined as percentage ratio between the copy number of target Chloroflexi 16S rRNA genes and the copy number of total bacterial 16S rRNA genes obtained from the same template DNA.

\section{Additional files}

Additional file 1: Table S1. PCB total concentration and congeners distribution of the samples used in this study.

Additional file 2: Figure S1. Sulfate-reduction activities in dechlorinating and non-dechlorinating cultures throughout incubation.

Additional file 3: Figure S2. Methanogenic activities in dechlorinating and non-dechlorinating cultures throughout incubation.

Additional file 4: Table S2. Features of primer pairs designed in this study. The primers target specifically VLD-1 and VLD-2 $16 S$ rRNA genes. The table report sequence, melting temperatures and in silico specificity analyses.

\section{Authors' contributions}

ANu performed most of the experiments and carried out the data analyses and interpretation. ANe helped with microcosms set-up and DGGE imageanalysis. GZ contributed to the study design and data interpretation. GZ and ANu wrote the manuscript. GZ and FF conceived the study and participated in its coordination. All authors read and approved the final manuscript.

\section{Acknowledgements}

Not applicable.

\section{Competing interests}

The authors declare that they have no competing interests.

\section{Availability of data and materials}

The datasets on which the findings and conclusions of this article is based upon are all included in this manuscript and the Additional files 1, 2, 3 and 4 associated with it.

\section{Funding}

This study was financially supported by the European Commission through Grant Number 266473 (Ulixes Project).

\section{Publisher's Note}

Springer Nature remains neutral with regard to jurisdictional claims in published maps and institutional affiliations.

Received: 23 November 2016 Accepted: 14 July 2017

Published online: 24 July 2017

\section{References}

1. Beyer A, Biziuk M. Environmental fate and global distribution of polychlorinated biphenyls. In: Whitacre DM, editor. Reviews of environmental contamination and toxicol, Vol. 201. New York: Springer; 2009. p. 137-58. doi:10.1007/978-1-4419-0032-6_5.

2. Stockholm Convention. Listing of POPs in the Stockholm. 2004. p. 1-9. http://chm.pops.int/TheConvention/ThePOPs/ListingofPOPs/tabid/2509/ Default.aspx. Accessed Apr 2017.

3. Thomas P. Effects of Aroclor 1254 and cadmium on reproductive endocrine function and ovarian growth in atlantic croaker. Mar Environ Res. 1989;28:499-503. http://www.sciencedirect.com/science/article/ pii/0141113689902912.

4. Carpenter DO. Polychlorinated biphenyls (PCBs): routes of exposure and effects on human health. Rev Environ Health. 2006;21:1-24. http://www.degruyter.com/view/j/reveh.2006.21.1/reveh.2006.21.1.1/ reveh.2006.21.1.1.xml.

5. Wong CS, Mabury SA, Whittle DM, Backus SM, Teixeira C, DeVault DS, et al. Organochlorine compounds in lake superior: chiral polychlorinated biphenyls and biotransformation in the aquatic food web. Environ Sci Technol. 2004;38:84-92. doi:10.1021/es0346983.

6. Bedard DL, Pohl EA, Bailey JJ, Murphy A. Characterization of the PCB substrate range of microbial dechlorination process LP. Environ Sci Technol. 2005;39:6831-8.

7. Wiegel J, Wu Q. Microbial reductive dehalogenation of polychlorinated biphenyls. FEMS Microbiol Ecol. 2000;32:1-15.

8. Khan Fl, Husain T, Hejazi R. An overview and analysis of site remediation technologies. J Environ Manag. 2004;71:95-122.

9. US EPA. Contaminated sediment remediation guidance for hazardous waste sites chapter 3 , feasibility study considerations. Office of solid waste emergency and response; 2005. p. 1-26. http://scholar.google. $\mathrm{com} / \mathrm{scholar}$ hl=en\&btnG=Search\&q=intitle:Contaminated+Sediment +Remediation+Guidance+for+Hazardous+Waste+Sites\#0. Accessed 9 Oct 2014.

10. Bedard DL. A case study for microbial biodegradation: anaerobic bacterial reductive dechlorination of polychlorinated biphenyls - from sediment to defined medium. Annu Rev Microbiol. 2008;62:253-70. 
11. Zhen H, Du S, Rodenburg LA, Mainelis G, Fennell DE. Reductive dechlorination of 1,2,3,7,8-pentachlorodibenzo-p-dioxin and Aroclor 1260, 1254 and 1242 by a mixed culture containing Dehalococcoides mccartyi strain 195. Water Res. 2014;52:51-62. http://linkinghub.elsevier.com/retrieve/ pii/S0043135414000049.

12. Kaya D, Imamoglu I, Sanin FD, Payne RB, Sowers KR. Potential risk reduction of Aroclor 1254 by microbial dechlorination in anaerobic grasse river sediment microcosms. J Hazard Mater. 2017;321:879-87. http://www. sciencedirect.com/science/article/pii/S0304389416309062.

13. Kjellerup BV, Naff C, Edwards SJ, Ghosh U, Baker JE, Sowers KR. Effects of activated carbon on reductive dechlorination of PCBs by organohalide respiring bacteria indigenous to sediments. Water Res. 2014;52:1-10. http://linkinghub.elsevier.com/retrieve/pii/S0043135413010294.

14. Wang S, He J. Phylogenetically distinct bacteria involve extensive dechlorination of Aroclor 1260 in sediment-free cultures. PLOS ONE. 2013;8:e59178.

15. Dudková V, Demnerová K, Bedard DL. Sediment-free anaerobic microbial enrichments with novel dechlorinating activity against highly chlorinated commercial PCBs. J Chem Technol Biotechnol. 2012;87:1254-62. doi:10.1002/jctb.3807.

16. He J, Bedard DL. The microbiology of anaerobic PCB dechlorination. In: Adrian L, Löffler FE, editors. Organohalide-respiring bact. Berlin: Springer; 2016. p. 541-62. doi:10.1007/978-3-662-49875-0.

17. Zanaroli G, Negroni A, Häggblom MM, Fava F. Microbial dehalogenation of organohalides in marine and estuarine environments. Curr Opin Biotechnol. 2015;33:287-95. http://linkinghub.elsevier.com/retrieve/pii/ S0958166915000579.

18. Bedard DL, Bailey JJ, Reiss BL, Jerzak GVS. Development and characterization of stable sediment-free anaerobic bacterial enrichment cultures that dechlorinate Aroclor 1260. Appl Environ Microbiol. 2006;72:2460-70. doi:10.1128/AEM.72.4.2460-2470.2006.

19. Adrian L, Dudkova V, Demnerova K, Bedard DL. "Dehalococcoides" sp. strain CBDB1 extensively dechlorinates the commercial polychlorinated biphenyl mixture Aroclor 1260. Appl Environ Microbiol. 2009;75:4516-24. http://www.pubmedcentral.nih.gov/articlerender.fcgi?artid=2704801\&to ol=pmcentrez\&rendertype $=$ abstract.

20. Bedard DL, Ritalahti KM, Loffler FE. The Dehalococcoides population in sediment-free mixed cultures metabolically dechlorinates the commercial polychlorinated biphenyl mixture Aroclor 1260. Appl Environ Microbiol. 2007;73:2513-21. http://www.pubmedcentral.nih.gov/articlerender. fcgi?artid=1855590\&tool=pmcentrez\&rendertype=abstract.

21. Fagervold SK, Watts JEM, May HD, Sowers KR. Sequential reductive dechlorination of meta-chlorinated polychlorinated biphenyl congeners in sediment microcosms by two different chloroflexi phylotypes. Appl Environ Microbiol. 2005;71:8085-90. http://aem.asm.org/content/71/12/8085.short.

22. Fagervold SK, May HD, Sowers KR. Microbial reductive dechlorination of Aroclor 1260 in Baltimore harbor sediment microcosms is catalyzed by three phylotypes within the phylum Chloroflexi. Appl Environ Microbiol. 2007;73:3009-18. http://www.pubmedcentral.nih.gov/articlerender.fcgi?a rtid $=1892865 \&$ tool=pmcentrez\&rendertype $=$ abstract.

23. LA Cutter, Watts JEM, Sowers KR, May HD. Identification of a microorganism that links its growth to the reductive dechlorination of 2,3,5,6-chlorobiphenyl. Environ Microbiol. 2001;3:699-709.

24. May HD, Miller GS, Kjellerup BV, Sowers KR. Dehalorespiration with polychlorinated biphenyls by an anaerobic ultramicrobacterium. Appl Environ Microbiol. 2008;74:2089-94.

25. Wu Q, Watts JEM, Sowers KR, May HD. Identification of a bacterium that specifically catalyzes the reductive dechlorination of polychlorinated biphenyls with doubly flanked chlorines. Appl Environ Microbiol. 2002;68:807-12. http://aem.asm.org/content/68/2/807.short.

26. Capone DG, Kiene RP. Comparison of microbial dynamics in marine and freshwater sediments: contrasts in anaerobic carbon catabolism. Limno Oceanogr. 1988;33:725-49. http://www.aslo.org/lo/toc/vol_33/issue_4_ part_2/0725.html.

27. Wang S, Chng KR, Chen C, Bedard DL, He J. Genomic characterization of Dehalococcoides mccartyi strain JNA that reductively dechlorinates tetrachloroethene and polychlorinated biphenyls. Environ Sci Technol. 2015;49:14319-25. doi:10.1021/acs.est.5b01979.

28. LaRoe SL, Fricker AD, Bedard DL. Dehalococcoides mccartyi strain JNA in pure culture extensively dechlorinates Aroclor 1260 according to polychlorinated biphenyl (PCB) dechlorination process N. Environ Sci Technol. 2014;48:9187-96.

29. Fricker AD, Laroe SL, Shea ME, Bedard DL. Dehalococcoides mccartyi strain JNA dechlorinates multiple chlorinated phenols including pentachlorophenol and harbors at least 19 reductive dehalogenase homologous genes. Environ Sci Technol. 2014;48:14300-8.

30. Wang S, Chng KR, Wilm A, Zhao S, Yang K-L, Nagarajan N, et al. Genomic characterization of three unique Dehalococcoides that respire on persistent polychlorinated biphenyls. Proc Natl Acad Sci USA. 2014;111:12103-8.

31. May HD, Sowers KR. "Dehalobium chlorocoercia" DF-1 - from DISCOVERY to application. Organohalide-respiring bact. Berlin: Springer; 2016. p. 563-86. doi:10.1007/978-3-662-49875-0.

32. Fava F, Gentilucci S, Zanaroli G. Anaerobic biodegradation of weathered polychlorinated biphenyls (PCBs) in contaminated sediments of Porto Marghera (Venice Lagoon, Italy). Chemosphere. 2003;53:101-9. http:// www.sciencedirect.com/science/article/pii/S0045653503004387.

33. Fava F, Zanaroli G, Young LY. Microbial reductive dechlorination of preexisting PCBs and spiked 2,3,4,5,6-pentachlorobiphenyl in anaerobic slurries of a contaminated sediment of Venice Lagoon (Italy). FEMS Microbiol Ecol. 2003;44:309-18. http://www.sciencedirect.com/science/article/pii/ S0168649603000692.

34. Zanaroli G, Balloi A, Negroni A, Borruso L, Daffonchio D, Fava F. A Chloroflexi bacterium dechlorinates polychlorinated biphenyls in marine sediments under in situ-like biogeochemical conditions. J Hazard Mater. 2012;209-210:449-57.

35. Zanaroli G, Pérez-Jiménez JR, Young LY, Marchetti L, Fava F. Microbial reductive dechlorination of weathered and exogenous co-planar polychlorinated biphenyls (pcbs) in an anaerobic sediment of Venice Lagoon. Biodegradation. 2006;17:19-27.

36. Oefjord GD, Puhakka JA, Ferguson JF. Reductive dechlorination of Aroclor 1254 by marine sediment cultures. Environ Sci Technol. 1994;28:2286-94. doi:10.1021/es00062a012.

37. Alder AC, Haggblom MM, Oppenheimer SR, Young LY. Reductive dechlorination of polychlorinated biphenyls in anaerobic sediments. Environ Sci Technol. 1993;27:530-8. doi:10.1021/es00040a012.

38. Matturro B, Ubaldi C, Grenni P, Caracciolo AB, Rossetti S. Polychlorinated biphenyl $(P C B)$ anaerobic degradation in marine sediments: microcosm study and role of autochthonous microbial communities. Environ Sci Pollut Res. 2016;23:12613-23. doi:10.1007/s11356-015-4960-2.

39. Wang $Y$, Sheng H-F, He Y, Wu J-Y, Jiang Y-X, Tam NF-Y, et al. Comparison of the levels of bacterial diversity in freshwater, intertidal wetland, and marine sediments by using millions of illumina tags. Appl Environ Microbiol. 2012;78:8264-71. http://aem.asm.org/cgi/content/long/78/23/8264.

40. Matturro B, Di Lenola M, Ubaldi C, Rossetti S. First evidence on the occurrence and dynamics of Dehalococcoides mccartyi PCB-dechlorinase genes in marine sediment during Aroclor 1254 reductive dechlorination. Mar Pollut Bull. 2016;112:189-94.

41. Matturro B, Ubaldi C, Rossetti S. Microbiome dynamics of a polychlorobiphenyl (PCB) historically contaminated marine sediment under conditions promoting reductive dechlorination. Front Microbiol. 2016;7:1-14.

42. Zanaroli G, Balloi A, Negroni A, Daffonchio D, Young LY, Fava F. Characterization of the microbial community from the marine sediment of the Venice Lagoon capable of reductive dechlorination of coplanar polychlorinated biphenyls (PCBs). J Hazard Mater. 2010;178:417-26.

43. Zanaroli G, Negroni A, Vignola M, Nuzzo A, Shu H-Y, Fava F. Enhancement of microbial reductive dechlorination of polychlorinated biphenyls (PCBs) in a marine sediment by nanoscale zerovalent iron (NZVI) particles. J Chem Technol Biotechnol. 2012;87:1246-53. doi:10.1002/jctb.3835.

44. Ho CH, Liu SM. Effect of coplanar PCB concentration on dechlorinating microbial communities and dechlorination in estuarine sediments. Chemosphere. 2011:82:48-55.

45. Kjellerup BV, Sun X, Ghosh U, May HD, Sowers KR. Site-specific microbial communities in three PCB-impacted sediments are associated with different in situ dechlorinating activities. Environ Microbiol. 2008;10:1296-309.

46. Kittelmann S, Friedrich MW. Novel uncultured Chloroflexi dechlorinate perchloroethene to trans-dichloroethene in tidal flat sediments. Environ Microbiol. 2008;10:1557-70.

47. Watts JEM. A PCR-based specific assay reveals a population of bacteria within the Chloroflexi associated with the reductive dehalogenation of polychlorinated biphenyls. Microbiology. 2005;151:2039-46. doi:10.1099/ mic.0.27819-0. 
48. Frame GM. A collaborative study of 209 PCB congeners and 6 Aroclors on 20 different HRGC columns. 2 Semi-quantitative Aroclor congener distributions. Fresenius J Anal Chem. 1997;357:714-22. doi:10.1007/ s002160050237.

49. Scoma A, Bertin L, Zanaroli G, Fraraccio S, Fava F. A physicochemicalbiotechnological approach for an integrated valorization of olive mill wastewater. Bioresour Technol. 2011;102:10273-9.

50. Marzorati M, Wittebolle L, Boon N, Daffonchio D, Verstraete W. How to get more out of molecular fingerprints: practical tools for microbial ecology. Environ Microbiol. 2008;10:1571-81.

51. Wittebolle L, Marzorati M, Clement L, Balloi A, Daffonchio D, Heylen K, et al. Initial community evenness favours functionality under selective stress. Nature. 2009:458:623-6.
52. Read S, Marzorati M, Guimarães BCM, Boon N. Microbial resource management revisited: successful parameters and new concepts. Appl Microbiol Biotechnol. 2011;90:861-71.

53. Ye J, Coulouris G, Zaretskaya I, Cutcutache I, Rozen S, Madden TL. PrimerBLAST: a tool to design target-specific primers for polymerase chain reaction. BMC Bioinform. 2012;13:134.

54. Yamada Y, Katsura K, Kawasaki H, Widyastuti Y, Saono S, Seki T, et al. Asaia bogorensis gen. nov., sp. nov., an unusual acetic acid bacterium in the alpha-Proteobacteria. Int J Syst Evol Microbiol. 2000;50:823-9.

55. Bach H-J, Tomanova J, Schloter M, Munch J. Enumeration of total bacteria and bacteria with genes for proteolytic activity in pure cultures and in environmental samples by quantitative PCR mediated amplification. J Microbiol Methods. 2002:49:235-45.

\section{Submit your next manuscript to BioMed Central and we will help you at every step:}

- We accept pre-submission inquiries

- Our selector tool helps you to find the most relevant journal

- We provide round the clock customer support

- Convenient online submission

- Thorough peer review

- Inclusion in PubMed and all major indexing services

- Maximum visibility for your research

Submit your manuscript at www biomedcentral com/submit 